\title{
Neuropilates to Improve Motor Function in Stroke: Past, present, and Future
}

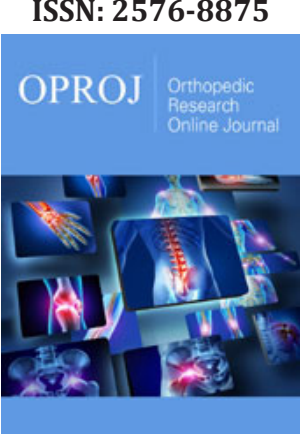

*Corresponding author: Eimear Cronin, Institute of Technology (IT) Sligo, Ireland

Submission: 眥 February 21, 2020

Published: 佂March 02, 2020

Volume 7 - Issue 1

How to cite this article: Eimear Cronin, David Roberts, Kenneth Monaghan. Neuropilates to Improve Motor Function in Stroke: Past, present, and Future. Ortho Res Online J. 7(1). OPROJ.000651.2020. DOI: 10.31031/OPROJ.2020.07.000651

Copyright@: Eimear Cronin, This article is distributed under the terms of the Creative Commons Attribution 4.0 International License, which permits unrestricted use and redistribution provided that the original author and source are credited.

\author{
Eimear Cronin ${ }^{1,2,3 *}$, David Roberts ${ }^{1,2}$ and Kenneth Monaghan ${ }^{1,2}$ \\ ${ }^{1}$ Clinical Health and Nutrition Centre (CHANCE), Institute of Technology (IT) Sligo, Ireland \\ ${ }^{2}$ Neuroplasticity Research Group, Clinical Health and Nutrition Centre (CHANCE), Ireland \\ ${ }^{3}$ HSE Physiotherapy Department, Ireland
}

Keywords: Neurological pilates; Neuropilates; Stroke; Rehabilitation

\section{Mini Review \\ Introduction to pilates}

Pilates as an exercise form was created by Joseph Pilates in the 1920s and is a programme of mind-body exercise focussing on strength, core stability, flexibility, muscle control, posture and breathing [1]. Joseph Pilates initially started using his exercises to rehabilitate bedridden world war 1 soldiers, often using the springs of the beds as resistance equipment [2]. In the late 1930s, the exercise method then became popular amongst the dance community benefitting them by improving performance and fitness and reducing time spent recovering from injuries [2]. The pilates method of exercise may have suited dancers above other forms of exercise due to the focus on alignment, core strength and flexibility and the aim of achieving neutral postures [3] and building long lean muscles as opposed increasing muscle bulk It also may have helped dancers to increase their kinaesthetic awareness, due to the mind-body nature of this exercise method, thereby enhancing their dancing. As an exercise form, pilates was initially introduced into rehabilitation by therapists specialising in dance medicine [2]. In more recent years, it has become an important and effective rehabilitation tool in wider patient groups, including those with musculoskeletal and rheumatological conditions, particularly for the purposes of reducing pain and disability [4]. The pilates method contains 6 key principles: centreing, concentration, control, precision, flow and breathing [5].

\section{Pilates in the non-clinical population}

In a systematic review in 2011 of 16 randomised controlled trials including 727 participants overall, investigating the effects of pilates exercise in healthy individuals, strong evidence was found for improving flexibility and dynamic balance, while moderate evidence was found for improving muscular endurance [6]. It is worth noting, however, that control groups were inactive in 11 of these 16 randomised controlled trials and so placebo and hawthorne effects may have been relevant in the findings. The authors found contradictory results for strength, with pilates exercises improving hip, lower back and abdominal strength in 3 of the studies when compared with no exercise, but with no additional abdominal strength benefits found in one study when compared with a postural education group [7]. Differences in strength measurement, duration of intervention and level of supervision and instruction may account for these contradictory results between studies. In healthy children, a systematic review has shown that pilates appears to improve flexibility, strength and postural control [8] and in elderly populations, pilates has also been shown to have benefits with one systematic review of 10 studies demonstrating improvements in muscle strength, walking, activities of daily living, quality of life and dynamic balance [9].

\section{Clinical pilates}

In clinical populations, the effects of pilates on low back pain has been examined in detail. A cochrane review of 10 trials, including 510 participants, showed low to moderate quality evidence that pilates reduces pain and disability in participants with non-specific low back pain when compared to minimal intervention. The authors, however, could not provide any 
conclusive evidence that pilates is superior to other forms of exercise [10]. Further systematic reviews have shown improvements in pain, range of motion and fatigue in women with breast cancer [11]. Pilates has also been shown to significantly reduce depressive and anxiety symptoms and feelings of fatigue in a systematic review of 8 studies. Sample sizes in the studies selected for inclusion in this latter review were small and the quality of the non-active control conditions was variable, therefore, further studies would be needed to clarify if pilates, as a mind-body connection enhancing exercise programme, would have superiority over a general exercise programme in improving mental health outcomes. Pilates has not only been studied and clinically used by therapists in populations with pain and movement loss, but also in populations with specific disabilities as a result of a neurological condition [12].

\section{Neuropilates}

Neuropilates, that is, the practice pf clinical pilates in patients with a neurological condition, is theorised to have beneficial effects on strength, postural control, alignment, stability, balance, proprioception, coordination and gait in those with deficits due to a neurological condition through retraining low threshold activity of local muscles and decreasing over-active global muscles [13]. Most of the literature in this field centres around populations with Multiple Sclerosis. The evidence investigating pilates training in people with multiple sclerosis is conflicting with the largest of these studies, a multi-centre randomised control trial with 94 participants showing no significant improvements in 10-metre walk test scores after 12 weeks of pilates exercises compared with 12 weeks of relaxation [14]. However, two smaller randomised control trials demonstrated significant improvements in balance, mobility and strength $[15,16]$. A systematic review examining the effects of pilates in people with Parkinson's Disease presented some preliminary evidence that the exercise form may have positive impacts on fitness, balance and particularly lower-body function in this patient cohort [17]. However, the authors emphasized the need for randomised controlled trials with larger sample sizes to confirm these observations.

\section{Neuropilates in stroke: The Principles and links with neuroplasticity}

Neuropilates could theoretically benefit stroke patients in many of the ways described above, though the evidence base to support this is small. According to the American Heart Association / American Stroke Association, a stroke is characterized as a "neurological deficit attributed to an acute focal injury of the central nervous system by a vascular cause, including cerebral infarction, intracerebral haemorrhage, and subarachnoid haemorrhage, and is a major cause of disability and death worldwide" [18]. In Ireland, approximately 10,000 people have a stroke related event every year with upwards of 30,000 people living in the community with disabilities as a result of a stroke [19]. Stroke survivors are often left with mobility, strength and balance deficits that pre-dispose them to a sedentary lifestyle, which further increases the risk of falls and risk of further stroke, whilst also impacting negatively on their functional inde- pendence and participation [20]. Studies have shown that physical activity levels of community dwelling people with stroke remain lower than their age-matched counterparts [21,22] and even lower than other older adults with chronic health conditions [23] Stroke survivors were shown to have the highest proportion of inactivity $(27 \%)$ and lowest participation in other activities when compared with other chronic disease cohorts such as diabetes, respiratory diseases and degenerative neurological disorders [23]. An Irish study by Horgan et al, found that $60 \%$ of stroke survivors questioned felt that their engagement in leisure activities had changed significantly after their stroke. Most of the affected leisure activities mentioned were physical including walking, swimming, cycling and going to the gym amongst others [24].

The benefits derived from pilates exercise of balanced strength with improved alignment, postural control and flexibility, could be favourable to stroke patients who often have unilateral loss of strength and muscle length and subsequent adverse postural adaptations and abnormal movement patterns. However, the exercises would have to be adapted to meet their needs with postural and positional adaptations and the use of supportive equipment as necessary. Joseph Pilates originally called his method of exercising "Contrology" which refers to the coordination of the body and mind. In his book "Return to Life" [25], Joseph Pilates stated that "Contrology begins with mind control over muscles" and also emphasized the importance of keeping "your mind wholly concentrated on the exercises as you perform them". This focus on control and concentration during exercise are key principles of pilates and they link well with neuroplasticity concepts such as attention and visualisation, which can be critical modulators of plasticity [26]. This all points to the potential benefit of "Contrology" style pilates exercises to those with neural damage post stroke.

"Centreing" as a key principle of pilates could be particularly beneficial to promote positive neuroplastic changes in stroke patients, who often experience an altered sense of centre. It has been clinically established that biases in verticality perception occur often after a stroke and cause postural disorders [27]. Pilates with a consistent and repetitive focus on neutral postures, centreing and alignment may minimise compensatory patterns and improve postural control and verticality perception, although this has yet to be studied in the research.

Another key principle of pilates which may be beneficial to stroke survivors is the concept of breath awareness. Breath holding during exercise can be common in stroke survivors with increased bodily effort and concentration on new movements. However, this can negatively impact on muscle tone and muscle fatigue with reduced overall efficiency. Again, this has not yet been studied in the literature but pilates may improve muscular efficiency and have positive impacts on tone by teaching patients how to breath appropriately and control their movements with maximum efficiency.

Further key principles of pilates are precision and flow. Precision relates to the importance of completing each exercise with 
preciseness and sustained attention to the alignment of each body part. In this way, it is most important in pilates training to complete the correct exercise with correct form and posture. Precision is an important principle in post stroke rehabilitation as it may improve concentration on and visualisation of correct movements of the affected limb and reduce compensatory strategies on the unaffected side. The number of repetitions a patient does is an important issue for functional improvement and cortical reorganisation in neuroplasticity [28], and a well taught patient who has gained some precision in their pilates exercises may be more likely to repeat the correct movement with correct form multiple times at home, unsupervised. Flow relates to the fluidity of movement, with the aim in pilates being smooth, continuous, flowing movements. Again, this focus may be beneficial in helping the post stroke patient to overcome dyskinesias or ataxic movements and aspiring toward graceful, coordinated movements, although this has yet to be researched.

\section{Neuropilates in stroke: The evidence}

Considering these pilates key principles and their potential contribution to neuroplastic and functional improvements in post stroke populations, it is important now to focus on what research has actually been completed in this field. A Cochrane review in 2016 [29] examined physical fitness training for stroke patients. After reviewing 58 trials, the authors concluded that cardiorespiratory training and to a lesser extent, mixed training could improve mobility and balance and that the evidence was robust enough to promote incorporating physical fitness training into post stroke rehabilitation programmes. However, pilates, as an exercise form, despite its ever-growing popularity in healthy individuals and potential benefits to post stroke patients, was not mentioned in this large Cochrane review.

A systematic review in 2018 examining pilates in noncommunicable diseases, which included stroke, and found strong evidence for improvement in exercise tolerance and moderate evidence for improvement in symptoms, muscle strength and quality of life [30]. The authors concluded that the most appropriate pilates protocol for each non-communicable disease was yet to be ascertained, but there was a trend towards longer interventions with additional components, such as education sessions or home exercise, at least three times per week being more effective. However, of the 12 studies reviewed, only two investigated pilates in chronic stroke patients and so overall results may not correlate to stroke patients exclusively and further high-quality evidence is needed.

Of the two studies mentioned in the above systematic review, one study [31] was given a global quality rating of 2 (moderate) by the authors. This small-scale Korean study provided some initial evidence that an 8-week pilates exercise group could significantly improve balance in chronic stroke patients when compared to a control group of no treatment in a randomised controlled trial of 19 participants. Balance was measured in this study by calculating the degree of centre of pressure sway during treadmill walking, which was significantly reduced in the intervention group. No standardised outcome measures were used to assess balance and the authors did recognise that follow up studies should be performed to investigate the impact of pilates training on gait parametres or lower limb EMG activity related to balance measures. The authors also made some suggestions for the conducting of a pilates exercise class in this population including limiting class numbers to 8 and an employing an 8-week training period, however these suggestions should be considered with caution in the stroke population as they were not based on the study at hand, but rather previous studies which had investigated pilates in healthy middle-aged women and healthy elderly participants.

The other study in the afore mentioned systematic review [32] was rated as "strong" by Miranda and Marques, 2018. This study found statistical improvements in cardiopulmonary function of chronic stroke patients, as measured by graded, submaximal treadmill exercise testing in a randomised control trial of 20 participants. The intervention group partook in 24 pilates sessions compared with a control group who partook in conventional stroke rehabilitation. Unlike the previous study, this study employed the use of a standardised outcome measure, "Timed up and Go" to assess gait and balance. The pilates group showed significant improvements in this test after 8 weeks of exercise. The authors concluded that pilates may have improved cardiopulmonary function in this study in a similar way to any regular aerobic exercise, through the maintenance of motor unit recruitment and muscle mass. However, they discussed the potential additional benefits of the focus on breathing in pilates exercises and how this may allow for easier performance of movements with less strain and improve lung capacity. The authors then linked this improvement in respiratory function with improved mobility function in the "Timed Up and Go" test.

A further randomised controlled trial, which also used the same, small participant numbers and the same intervention period of pilates training ( 3 times a week for 8 weeks) showed significant improvements in gait parameters post pilates training in the intervention group [33]. Here, gait was measured with a 3-D motion analysis with 8 infrared cameras. One gait parameter which did not improve significantly in the intervention group was the gait asymmetry index and the authors suggested employing a longer exercise period in future studies to assess if gait symmetry could be statistically improved through pilates exercising.

All three of these studies were conducted in Korea with similar exercise protocols, participant numbers and methodology. The only study outside of Korea on this topic was an American case report [34] to document the feasibility of using pilates in one individual with a stroke. The participant was instructed in pilates exercises twice weekly for nine months. Improvements were found in balance, strength and quality of life, but not in posture or gait speed. The authors could only infer the possible benefit of pilates as an addition to his overall programme, but no firm conclusions could be drawn. However, the authors did comment that pilates, with some 
modifications was a feasible form of exercise to use in someone with a stroke.

So, what can this handful of small-scale studies reliably tell us? They point to the feasibility and possible benefits of pilates training in post stroke patients but more robust, randomised clinical trials with larger participant numbers, utilising standardised outcome measures are needed to clarify the effectiveness of pilates on outcomes in this patient group. No study has investigated the effects of pilates on strength, spasticity, disability or functional independence in chronic stroke patients. Also, further research is needed to clarify what intensity, intervention protocol, pilates equipment and exercise adaptations would result in the best outcomes.

\section{Conclusion and Future Research}

Our research group is planning a clinical trial to investigate group (supervised) pilates training compared with an individualised, unsupervised home exercise pilates programme. We will examine the effects of both supervised and unsupervised pilates training on gait, function, independence, spasticity and strength in chronic stroke patients in a randomised controlled trial. In this way, we aim to add to the literature base by providing more information on the types, quantities and quality of pilates training in chronic stroke patients and by examining the effects of this training on formerly sparsely studied outcomes. We feel that widening the literature base in this way and providing pilates classes and education in the community is one step in the right direction toward providing evidence based, accessible and local exercise programmes for post stroke patients. This may assist with early supported discharge programmes, shortening a patient's rehabilitation stay, whilst also improving long term outcomes and encouraging a culture of exercise in the mostly sedentary post stroke population.

\section{References}

1. Wells C, Kolt GS, Bialocerkowski A (2012) Defining Pilates exercise: A systematic review. Complement Ther Med 20(4): 253-262.

2. Owsley A (2005) An introduction to clinical pilates. International Journal of Athletic Therapy and Training 10(4): 19-25.

3. Ahearn EL, Greene A, Lasner A (2018) Some effects of supplemental pilates training on the posture, strength, and flexibility of dancers 17 to 22 years of age. J Dance Med Sci22(4): 192-202.

4. Byrnes K, Wu PJ, Whillier S (2018) Is pilates an effective rehabilitation tool? A systematic review. Journal of Bodywork and Movement Therapies 22(1): 192-202.

5. Latey P (2001) The pilates method: history and philosophy. J Bodyw Mov Ther 5(4): 275-282.

6. Cruz-Ferreira A, Fernandes J, Laranjo L, Bernardo LM, Silva A (2011) A systematic review of the effects of Pilates method of exercise in healthy people. Arch Phys Med Rehabil 92(12): 2071-2081.

7. Donahoe-Fillmore B, Hanahan N, Mescher M, Clapp D, Addison N, et al. (2007) The effects of a home Pilates program on muscle performance and posture in healthy females: a pilot study. J Womens Health Phys Ther 31: 6-11.
8. Hornsby E, Johnston LM (2019) Effect of pilates intervention on physical function of children and youth: A systematic review. Arch Phys Med Rehabil 101(2): 317-328.

9. Bullo V, Bergamin M, Gobbo S, Sieverdes JC, Zaccaria M, et al. (2015) The effects of pilates exercise training on physical fitness and wellbeing in the elderly: A systematic review for future exercise prescription. Prev Med 75: 1-11.

10. Yamato TP, Maher CG, Saragiotto BT, Hancock MJ, Ostelo RW, et al. (2016) Pilates for low back pain: Complete Republication of a cochrane review. Spine 41(12): 1013-1021.

11. Espíndula RC, Nadas GB, Rosa MID, Foster C, Araújo FC, et al. (1992) Pilates for breast cancer: A systematic review and meta-analysis. Rev Assoc Med Bras 63(11): 1006-1012.

12. Fleming KM, Herring MP (2018) The effects of pilates on mental health outcomes: A meta-analysis of controlled trials. Complement Ther Med 37: 80-95.

13. Withers G (2009) Modified pilates rehabilitation programme (manual) pilates and neurology.

14. Fox EE, Hough AD, Creanor S, Gear M, Freeman JA (2016) Effects of pilates-based core stability training in ambulant people with multiple sclerosis: Multicenter, assessor-blinded, randomized controlled trial. Phys Ther 96(8): 1170-1178.

15. Guclu-Gunduz A, Citaker S, Irkec C, Nazliel B, Batur-Caglayan HZ (2014) The effects of pilates on balance, mobility and strength in patients with multiple sclerosis. Neuro Rehabilitation 34(2): 337-342.

16. Bulguroglu I, Guclu-Gunduz A, Yazici G, Ozkul C, Irkec C, et al. (2017) The effects of mat pilates and reformer pilates in patients with multiple sclerosis: A randomized controlled study. Neuro Rehabilitation 41(2): 413-422.

17. Suárez-Iglesias D, Miller KJ, Seijo-Martínez M, Ayán C (2019) Benefits of pilates in parkinson's disease: A systematic review and meta-analysis. Medicina (Kaunas) 55(8).

18. Sacco RL, Kasner SE, Broderick JP, Caplan LR, Connors JJ, et al. (2013) An updated definition of stroke for the $21^{\text {st }}$ century: a statement for healthcare professionals from the American Heart Association/ American Stroke Association. Stroke 44(7): 2064-2089.

19. McElwaine P, McCormack J, Harbison J (2016) On behalf of the national stroke programme Irish heart foundation/HSE national stroke audit rehabilitation units 2016.

20. Billinger SA, Arena R, Bernhardt J, Eng JJ, Franklin BA, et al. (2014) Physical activity and exercise recommendations for stroke survivors: a statement for healthcare professionals from the American Heart Association/American Stroke Association. Stroke 45: 2532-2553.

21. English C, Healy GN, Coates A, Lewis L, Olds T, et al. (2016) Sitting and activity time in people with stroke. Physical Activity 96(2): 193-201.

22. Tieges Z, Mead G, Allerhand M (2015) Sedentary behavior in the first year after stroke: a longitudinal cohort study with objective measures. Arch Phys Med Rehabil 96(1): 15-23.

23. Ashe MC, Miller WC, Eng JJ, Noreau L (2009) Older adults, chronic disease and leisure-time physical activity. Gerontology 55: 64-72.

24. Horgan F, Walsh M, Galvin R, Macey C, Loughrane C (2014) Experiences and long-term needs reported by stroke survivors living in the community in Ireland.

25. Pilates JH, Miller WJ (1960) Return to life through Contrology.

26. Cramer SC, Sur M, Dobkin BH, O’Brien C, Sanger TD, et al. (2011) Harnessing neuroplasticity for clinical applications. Brain 134(Pt 6): 1591-1609. 
27. Pérennou D, Piscicelli C, Barbieri G, Jaeger M, Marquer A, et al. (2014) Measuring verticality perception after stroke: why and how? Neurophysiol Clin 44(1): 25-32.

28. Kimberley TJ, Samargia S, Moore LG, Shakya JK, Lang CE (2010) Comparison of amounts and types of practice during rehabilitation for traumatic brain injury and stroke. J Rehabil Res Dev 47(9): 851-862.

29. Saunders DH, Sanderson M, Hayes S, Kilrane M, Greig CA, et al. (2016) Physical fitness training for stroke patients. Cochrane Database Syst Rev 3: CD003316.

30. Miranda S, Marques A (2018) Pilates in noncommunicable diseases: A systematic review of its effects. Complement Ther Med 39: 114-130.

31. Lim HS, Kim YL, Lee SM (2016) The effects of pilates exercise training on static and dynamic balance in chronic stroke patients: a randomized controlled trial. J Phys Ther Sci 28(6): 1819-1824.
32. Lim HS, Yoon S (2017) The effects of pilates exercise on cardiopulmonary function in the chronic stroke patients: a randomized controlled trials. J Phys Ther Sci 29(5): 959-963.

33. Roh SY, Gil HJ, Yoon S (2016) Effects of 8 weeks of mat-based Pilates exercise on gait in chronic stroke patients. J Phys Ther Sci 28(9): 26152619.

34. Shea S, Moriello G (2014) Feasibility and outcomes of a classical Pilates program on lower extremity strength, posture, balance, gait, and quality of life in someone with impairments due to a stroke. J Bodyw Mov Ther18(3): 332-360. 